\title{
Stromal cell-derived factor-1 promotes human adipose tissue-derived stem cell survival and chronic wound healing
}

\author{
QIANG LI ${ }^{1,2^{*}}$, YANPING GUO ${ }^{1 *}$, FEIFEI CHEN ${ }^{3}$, JING LIU $^{1}$ and PEISHENG JIN ${ }^{1}$ \\ ${ }^{1}$ Plastic Surgery Department, Affiliated Hospital of Xuzhou Medical College, Xuzhou, Jiangsu 221002; \\ ${ }^{2}$ Department of Ear Reconstruction, Plastic Surgery Hospital, Chinese Academy of Medical Sciences, Beijing 100144; \\ ${ }^{3}$ Jiangsu Key Laboratory of Biological Cancer Therapy, Xuzhou Medical College, Xuzhou, Jiangsu 221002, P.R. China
}

Received November 22, 2014; Accepted February 4, 2015

DOI: $10.3892 / \mathrm{etm} .2016 .3309$

\begin{abstract}
Adipose tissue-derived stem cells (ADSCs) hold great potential for the stem cell-based therapy of cutaneous wound healing. Stromal cell-derived factor-1 (SDF-1) activates $\mathrm{CXC}$ chemokine receptor $(\mathrm{CXCR}) 4^{+}$and $\mathrm{CXCR} 7^{+}$cells and plays an important role in wound healing. Increasing evidence suggests a critical role for SDF-1 in cell apoptosis and the survival of mesenchymal stem cells. However, the function of SDF-1 in the apoptosis and wound healing ability of ADSCs is not well understood. The aim of this study was to analyze the effect of SDF-1 on the apoptosis and therapeutic effect of ADSCs in cutaneous chronic wounds in vitro and in vivo. By flow cytometric analysis, it was found that hypoxia and serum free promoted the apoptosis of ADSCs. When pretreated with SDF-1, the apoptosis of ADSCs induced by hypoxia and serum depletion was partly recovered. Furthermore, in vivo experiments established that the post-implantation cell survival and chronic wound healing ability of ADSCs were increased following pretreatment with SDF-1 in a diabetic mouse model of chronic wound healing. To explore the potential mechanism underlying the effect of SDF-1 on ADSC apoptosis, western blot analysis was employed and the results indicate that SDF-1 may protect against cell apoptosis in hypoxic and serum-free conditions through activation of the caspase signaling pathway in ADSCs. This study provides evidence that SDF-1 pretreatment can increase the therapeutic effect of ADSCs in cutaneous chronic wounds in vitro and in vivo.
\end{abstract}

Correspondence to: $\mathrm{Dr}$ Peisheng Jin, Plastic Surgery Department, Affiliated Hospital of Xuzhou Medical College, 99 West Huai-Hai Road, Xuzhou, Jiangsu 221002, P.R. China E-mail: fallin1983@163.com

*Contributed equally

Key words: adipose-derived stem cells, stromal cell-derived factor-1, apoptosis, chronic wound, wound healing

\section{Introduction}

Chronic wounds are open wounds of the skin taking longer than 8 weeks to heal. An impaired healing process is often associated with chronic wounds (1). As an ongoing challenge in advanced wound care, chronic wounds can lead to severe outcomes, including infection, amputation and even mortality (2). Evidence has emerged that adipose tissue-derived stem cells (ADSCs) hold great potential in the stem cell-based therapy of chronic wounds $(3,4)$. ADSCs, which are also referred to as adipose mesenchymal stromal cells or adipose tissue-derived stromal cells, are adult stem cells that are derived from adipose tissue stroma, possess considerable self-renewal ability and can differentiate into various types of functional cells. ADSCs show a greater ability to reconstitute hematopoiesis and repair tissue than bone marrow stromal stem cells $(5,6)$. However, a significant barrier to the successful use of mesenchymal stem cells (MSCs) in any potential cell therapy has been post-implantation cell survival in cutaneous chronic wound models (7). When cells are transplanted into damaged cutaneous tissue where they experience a harsh wound environment, including hypoxia, inflammation, oxidative stress or other adverse conditions, this inevitably leads to the poor survival of seed cells following implantation, which markedly disturbs the therapeutic effects of MSCs (8). Various strategies have been used to improve cell survival following implantation $(9,10)$. However, simple and efficient interventions are required to strengthen the resistance of cells to apoptosis and increase the therapeutic effect of ADSCs.

Stromal cell-derived factor-1 (SDF-1) is an important chemokine that plays a central role in tissue repair and angiogenesis. SDF-1 exerts its biological function by binding to two G-protein-coupled receptors: CXC chemokine receptor (CXCR)4 and CXCR7 (11). SDF-1 functions in the mobilization and recruitment of stem and progenitor cells, including hematopoietic stem cells, MSCs and other CXCR4-expressing cells $(11,12)$. Increasing evidence suggests that the interaction of SDF-1 with CXCR4 and CXCR7 is critical in maintaining the proliferation and survival of MSCs in response to stress $(13,14)$. Studies have confirmed that SDF-1 can enhance the proliferation, migration, secretion and anti-apoptotic capacity of MSCs and improve cell transplant survival (15-17). However, very little is known about the effect of SDF-1 on 
ADSC apoptosis and survival. The current authors have previously shown that the interactions of SDF-1 with CXCR4 and CXCR7 are important in the paracrine actions, proliferation and migration of ADSCs (18). In the present study, the function of SDF-1 as an apoptosis inhibitor in vitro and the survival rate and therapeutic effect of ADSCs administered to the chronic wounds of diabetic nude mice were investigated. To the best of our knowledge, this is the first study to investigate whether SDF-1 improves the ability of ADSCs to repair chronic wounds.

\section{Materials and methods}

ADSC culture. Human ADSCs were isolated from subcutaneous adipose tissue samples obtained from the liposuction aspirates of patients undergoing cosmetic liposuction as previously described (19). Three healthy female patients with abdominal fat accumulation who underwent liposuction surgery at the Affiliated Hospital of Xuzhou Medical College (Xuzhou, China) were selected. This study was performed using a protocol approved by the Institutional Review Board of the Affiliated Hospital of Xuzhou Medical College and all examinations were performed after obtaining written informed consent from the patients. ADSCs were maintained in L-Dulbecco's modified Eagle's medium (DMEM; Invitrogen Life Technologies, Carlsbad, CA, USA) containing $10 \%$ fetal bovine serum (FBS; Invitrogen Life Technologies). Cells were cultured in a $37^{\circ} \mathrm{C}$ humidified incubator with $95 \%$ air and $5 \% \mathrm{CO}_{2}$. Prior to experimental use, it was confirmed that the ADSCs possessed the ability to differentiate into osteoblasts and keratinocytes.

Hypoxic and serum-free conditions. ADSCs from the same passage were obtained from six flasks and randomly divided into three groups. These were the control group (L-DMEM containing $10 \%$ FBS, normoxic conditions), apoptosis model group (L-DMEM containing 1\% FBS, hypoxic conditions) and SDF-1 treatment group (L-DMEM containing 1\% FBS and $0.5 \mathrm{mg} / 1 \mathrm{SDF}-1)$. In the SDF-1 treatment group, ADSCs were initially cultured with L-DMEM containing $10 \%$ FBS and $0.5 \mathrm{mg} / 1$ SDF-1 (R\&D Systems, Minneapolis, MN, USA) in normoxic conditions for $1 \mathrm{~h}$. The cells were then rinsed twice with PBS and exposed to hypoxic and serum-free conditions with $0.5 \mathrm{mg} / \mathrm{l} \mathrm{SDF}-1$ for $6 \mathrm{~h}$. The apoptosis model and SDF-1 treatment group ADSCs were put into a hypoxic incubator (Forma Series II 3110 Water-Jacketed $\mathrm{CO}_{2}$ Incubator; Thermo Fisher Scientific, Waltham, MA, USA) and maintained in $1 \% \mathrm{O}_{2}$ to culture the cells under hypoxic conditions.

Western blot analysis. Following the treatments, total protein from the cells was extracted using radio-immunoprecipitation assay lysis buffer (Santa Cruz Biotechnology, Inc., Dallas, TX, USA) containing $60 \mu \mathrm{g} / \mathrm{ml}$ phenylmethylsulfonyl fluoride. Protein concentrations were assessed using a bicinchoninic acid protein assay kit (Wuhan Boster Biological Technology, Ltd., Wuhan, China). Proteins were separated on SDS-polyacrylamide gel, then transferred to a nitrocellulose membrane and incubated overnight at $4{ }^{\circ} \mathrm{C}$ with the following antibodies: Rabbit monoclonal anti-procaspase 3 (1:1,000; cat. no. 9665), anti-cleaved caspase 3 (1:1,000; cat. no. 9664), anti-poly ADP ribose polymerase (PARP; 1:1,000; cat. no. 9532) and anti-cleaved PARP (1:1,000; cat. no. 5625) and mouse monoclonal anti- $\beta$-actin (1:5,000; cat. no. 3700) (Cell Signaling Technology, Inc., Beverly, MA, USA). Blots were washed four times with TBS containing $0.1 \%$ Tween-20 (TBST), and then incubated with horseradish peroxidase (HRP)-linked horse anti-mouse and anti-rabbit secondary antibodies (1:200; cat. no. 7076 and 7074S respectively; Cell Signaling Technology, Inc.) at room temperature for $1 \mathrm{~h}$. Antibody binding was detected using a SuperSignal West Pico Chemiluminescent Substrate kit (Pierce Biotechnology, Inc., Rockford, IL, USA) according to the manufacturer's instructions.

Measurement of apoptosis by Annexin $V$ analysis. Following treatment, ADSCs were collected and dual staining with Annexin V-fluorescein isothiocyanate (FITC) and propidium iodide (PI) was performed according to the manufacturer's instructions (Nanjing KeyGen Biotech Co., Ltd., Nanjing, China). In brief, the collected cells were briefly washed with ice-cold phosphate-buffered saline (PBS) twice and resuspended in $200 \mu \mathrm{l} 1 \mathrm{X}$ binding buffer (Beyotime Institute of Biotechnology, Haimen, China) containing $5 \mu$ l Annexin V-FITC for $15 \mathrm{~min}$ followed by $300 \mu \mathrm{l} 1 \mathrm{X}$ binding buffer containing $5 \mu \mathrm{l} \mathrm{PI}$ for $5 \mathrm{~min}$ at room temperature in the dark. Following incubation, the cells were analyzed using a FACStar flow cytometer (BD Biosciences, Franklin Lakes, NJ, USA). An inverted fluorescence microscope (Olympus Corporation, Tokyo, Japan) was used to observe the cells in the early and late stages of apoptosis.

Dermal chronic wound model. The diabetic chronic skin wound model was established according to a previously described procedure with certain improvements (20). A total of 8 female mice were used that were housed with a constant humidity of $50-60 \%$ and a temperature of $22-24^{\circ} \mathrm{C}$ with a $12 \mathrm{~h} / 12 \mathrm{~h}$ light/dark cycle and access to food and water ad libitum.

Diabetes was induced in nude mice (Shanghai SLAC Laboratory Animal Co., Shanghai, China) by the intraperitoneal injection of streptozotocin (STZ; Sigma-Aldrich, St. Louis, MO, USA), dissolved in sodium citrate buffer (0.01 M; $\mathrm{pH} 4.5$ ). Control mice were treated with DMEM only. Blood glucose levels $>250 \mathrm{mg} / \mathrm{dl}$ in two consecutive measurements separated by an interval of $48 \mathrm{~h}$ were considered to indicate that the animals were diabetic. After anesthetizing the animals, a full thickness skin defect of 1-cm diameter was made on the back. The wound was covered with a sterile dressing, and 10 days later the granulation tissue was debrided, to re-establish a 1-cm-diameter wound. Following each surgery, 4,000 units penicillin sodium was injected intramuscularly to prevent infection.

ADSC survival assay in vivo. ADSCs were marked with 5-bromo-2'-deoxyuridine (5-BrdU; Sigma-Aldrich) prior to injection. Cells suspended in serum-free DMEM were treated with BrdU solution at a final concentration of $10 \mathrm{mmol} / \mathrm{l}$ at $37^{\circ} \mathrm{C}$ for $48 \mathrm{~h} .1 \times 10^{6} \mathrm{ADSCs}$ that were pretreated with $0.5 \mathrm{mg} / 1 \mathrm{SDF}-1$ or untreated were injected around the wound bed. The animals were sacrificed 7 days after treatment, and 

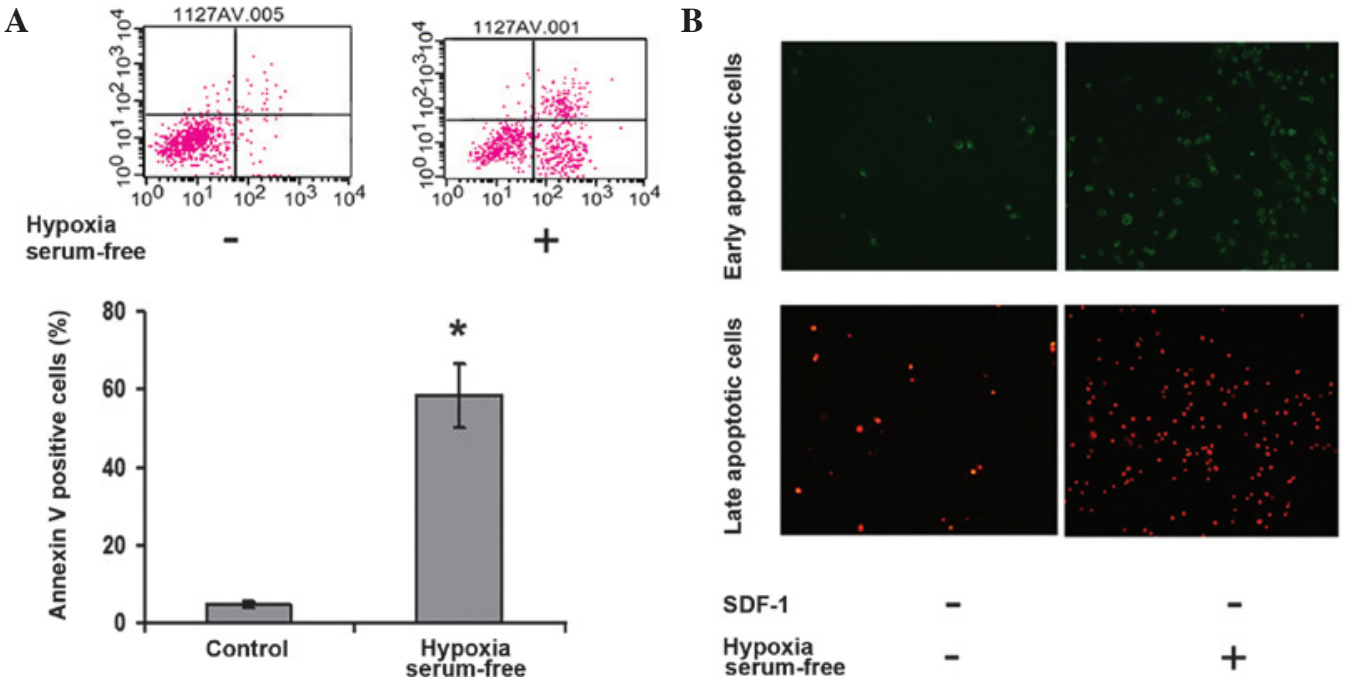

Figure 1. Hypoxia and serum-free conditions induce apoptosis in ADSCs. (A) ASDCs were stained with Annexin V-FITC/PI and immediately analyzed by flow cytometry. (B) Early and late apoptosis in ADSCs were detected by staining with Annexin V-FITC (green) and PI (red) under hypoxia and serum-free conditions. "P<0.05 vs. the control group. ADSC, adipose tissue-derived stem cell; FITC, fluorescein isothiocyanate; PI, propidium iodide.
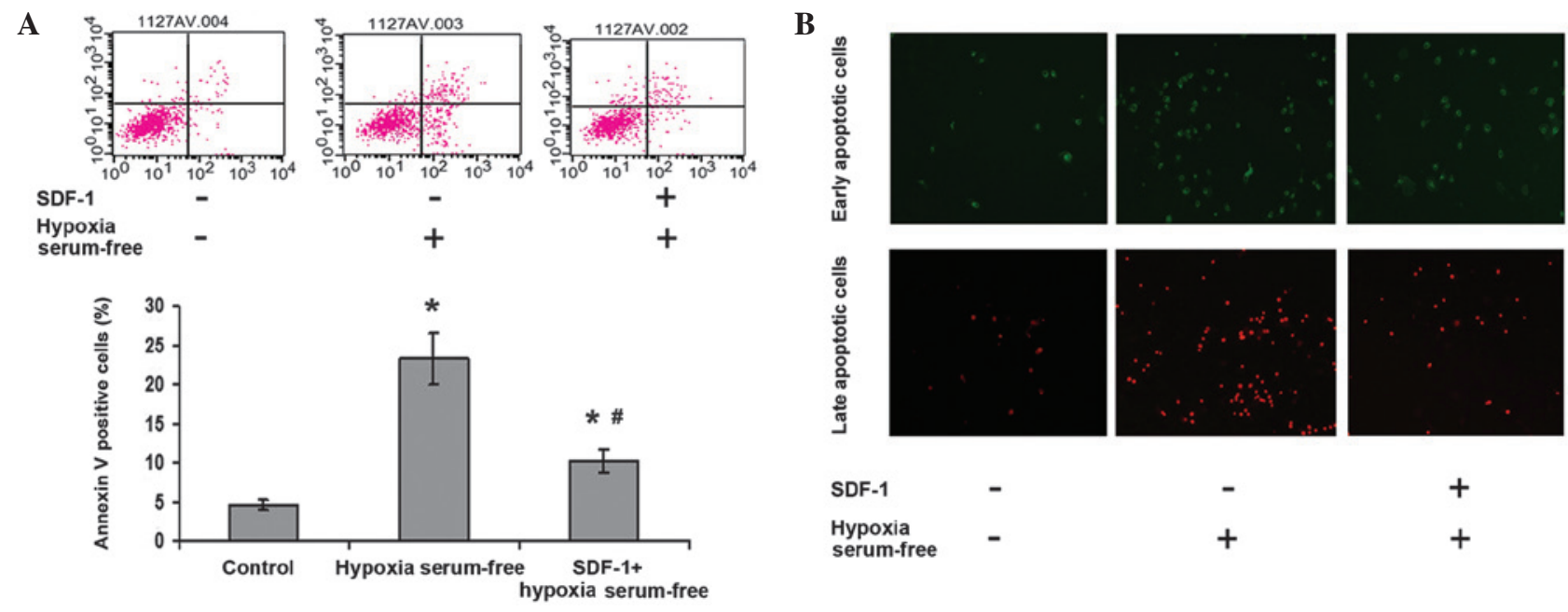

Figure 2. SDF-1 attenuated the apoptosis of ASDCs induced by hypoxic and serum-free conditions. (A) ASDCs were stained with Annexin V-FITC and PI and immediately analyzed by flow cytometry. (B) Effect of SDF-1 on the early and late apoptosis of ASDCs was detected by staining with Annexin V-FITC (green) and PI (red), respectively. "P<0.05 vs. the control group; ${ }^{\prime} \mathrm{P}<0.05$ vs. the hypoxia and serum-free group. SDF-1, stromal cell-derived factor-1; ASDC, adipose tissue-derived stem cell; FITC, fluorescein isothiocyanate; PI, propidium iodide.

wounds were harvested with the surrounding tissue. The specimens were fixed with $4 \%$ paraformaldehyde for $24 \mathrm{~h}$ and embedded in paraffin for histological sectioning. FITC immunofluorescence staining was performed according to the manufacturer's instructions (Beijing Zhongshan Golden Bridge Biotechnology Co., Ltd., Beijing, China); primary mouse monoclonal anti-BrdU antibodies (1:1,000; cat. no. sc-51514; Santa Cruz Biotechnology, Inc.) and FITC-labeled goat anti-mouse secondary antibodies (1:1,000; cat. no. sc-2010; Santa Cruz Biotechnology, Inc.) were added in sequence. Immunofluorescence microscopy was used, and the green fluorescence indicated 5-BrdU-labeled ADSC nuclei. Counting was conducted in 10 immunofluorescent images per group to determine the survival rate.

Cutaneous wound repair analysis. The animals were sacrificed by cervical dislocation 10 days after treatment. The wound area was analyzed by tracing the wound margin and calculating the area using ImageJ software (version 1.38; National Institutes of Health, Bethesda, MD, USA). The percentage of wound closure was calculated as follows: Wound closure index $(\%)=($ original wound area - unhealed wound area)/original wound area) x 100. Tissue specimens were embedded in paraffin for sectioning. The 4-mm sections were stained with hematoxylin and eosin. Using a light microscope, the re-epithelialization length of each specimen was measured from the marginal skin to the end of the neoepithelium on each side of the cross-section.

Statistical analysis. Data are expressed as mean \pm standard deviation and were statistically analyzed using a Student's t-test performed with SPSS software, version 11.5 (SPSS, Inc., Chicago, IL, USA). P $<0.05$ was considered to indicate a statistically significant difference. 
A

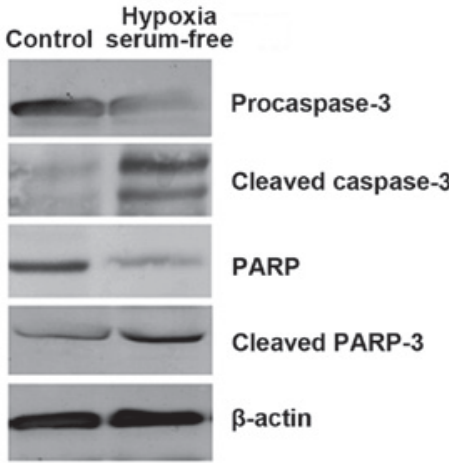

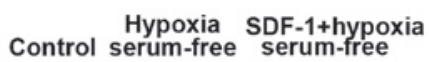

Control serum-free serum-free

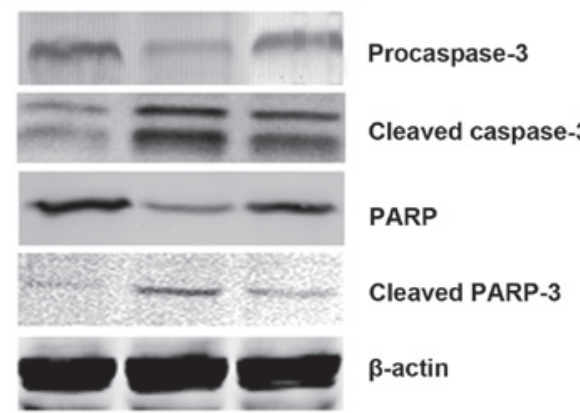

Figure 3. The caspase pathway is involved in the inhibitory effect of SDF-1 on the hypoxia and serum depletion-induced apoptosis of ADSCs. Western blot detection of the levels of procaspase-3, cleaved caspase-3, PARP and cleaved PARP following exposure to hypoxia and serum-free conditions for $6 \mathrm{~h}$ in (A) ADSCs and (B) ADSCs pretreated with SDF-1 (0.5 mg/l) for $2 \mathrm{~h}$. SDF-1, stromal cell-derived factor-1; ADSC, adipose tissue-derived stem cell; PARP, poly ADP ribose polymerase.

A

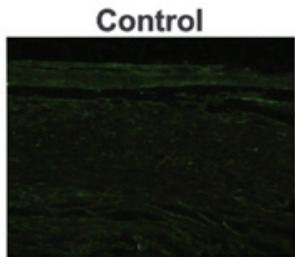

B

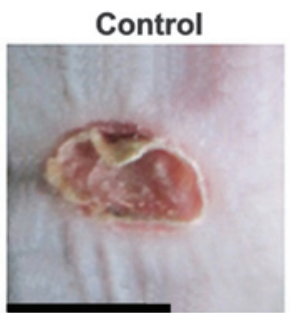

C

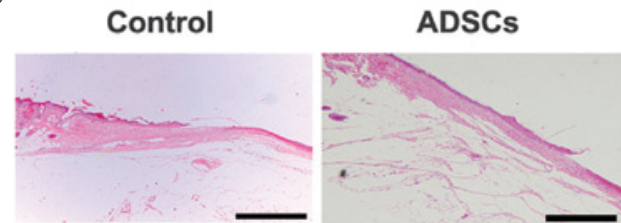

ADSCs

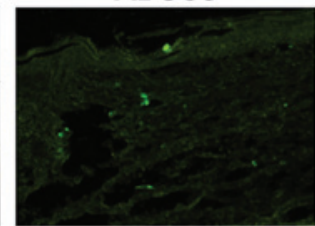

ADSCs
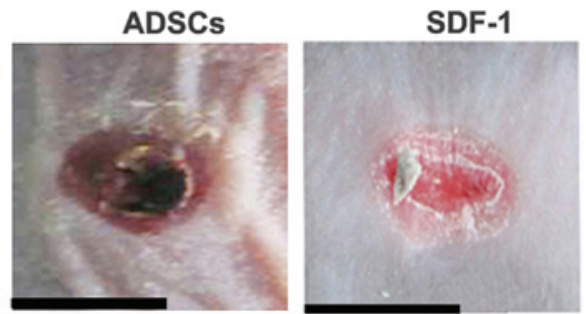

SDF-1
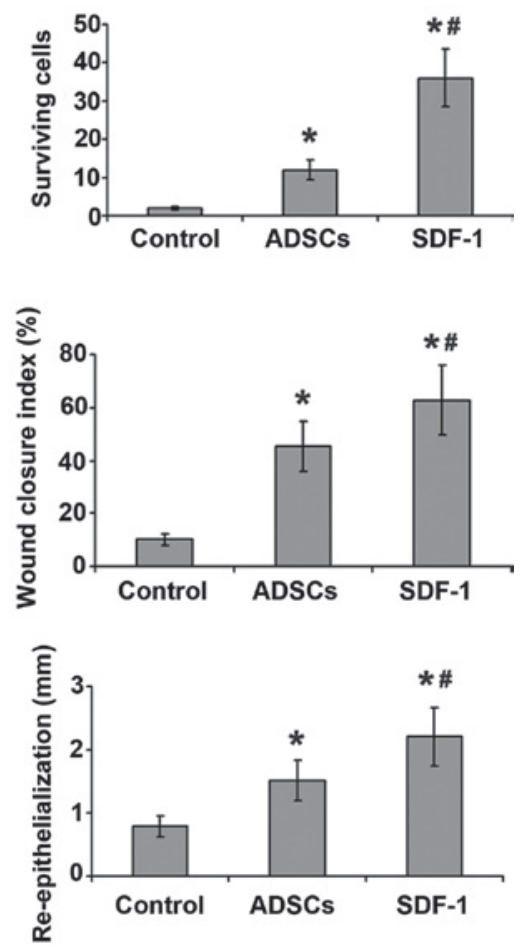

Figure 4. SDF-1 promotes ADSC survival and improves chronic wound healing in vivo. (A) ADSCs marked with 5-BrdU were injected into the chronic wounds; green fluorescence indicates 5-BrdU-labeled ADSCs nuclei. Immunofluorescence staining shows that only a few cells survived in the wound bed 7 days after transplantation in the ADSC group. The number of surviving cells significantly increased in the SDF-1-treated group. (B) Gross appearance of the skin wounds 10 days after treatment. In the SDF-1 group, wound areas were smaller, and the wound closure index was superior to that in the ASDC and control groups. (C) Histological results. H\&E staining of the wounds shows that SDF-treated ADSCs promote chronic wound repair, increase dermal collagen levels and the numbers of fibroblasts and capillaries, and result in an re-epithelization length superior to that in the ASDC and control groups. "P<0.05 vs. the control group; ${ }^{\#} \mathrm{P}<0.05$ vs. the ADSC group. SDF-1, stromal cell-derived factor-1; ADSC, adipose tissue-derived stem cell; 5-BrdU, 5-bromo-2'-deoxyuridine.

\section{Results}

Hypoxia and serum depletion induce apoptosis in ADSCs. To determine the effect of hypoxia and serum depletion on cell apoptosis, ADSCs were exposed to hypoxic and serum-free conditions for $6 \mathrm{~h}$. Flow cytometry was then performed using cell binding of the fluorescent dye Annexin V-FITC, which marks the early stage membrane change in apoptosis, and PI staining of the nucleus, which labels late apoptotic events and necrosis. As shown in Fig. 1, hypoxia and serum depletion evidently induced the number of apoptotic cells $(\mathrm{P}<0.05)$.
SDF-1 inhibits the hypoxia and serum depletion-induced apoptosis of ASDCs. To explore the effect of SDF-1 on the apoptosis of ADSCs induced by hypoxia and serum depletion, ADSCs were treated with SDF-1 prior to induction, then collected and analyzed by flow cytometry. As shown in Fig. 2, the number of apoptotic cells was increased in the hypoxia and serum depletion (apoptosis model) group compared with that in the control group $(\mathrm{P}<0.05)$; however, in the SDF-1 treatment group the numbers of early and late apoptotic cells were clearly decreased compared with those in the apoptosis model group. This suggests that SDF-1 
suppresses the apoptosis of ADSCs induced by hypoxia and serum depletion.

Caspase-3 and PARP activation are involved in the inhibitory effect of SDF-1 on the hypoxia and serum depletion-induced apoptosis of ASDCs. To further determine whether hypoxia and serum depletion-induced apoptosis is associated with activation of the caspase signaling pathway in ADSCs, caspase-3 and PARP were detected after induction. As shown in Fig. 3A, compared with that in the control group, the procaspase-3 level decreased following induction. By contrast, the cleaved caspase- 3 level increased compared with that in the control group. Similar changes were observed for PARP. However, when pretreated with SDF-1, procaspase-3 and PARP protein levels partly recovered and cleaved caspase- 3 and PARP protein levels decreased compared with those in the apoptosis model group. This shows that SDF-1 protects ADSCs from apoptosis under hypoxic and serum-free conditions by activating the caspase signaling pathway.

SDF-1 promotes ADSC survival in vivo. To investigate whether SDF-1 inhibits ASDC apoptosis in vivo, ADSCs marked with 5-Brd were injected into the chronic wounds of diabetic mice. It was found that only a few cells survived in the wound bed 7 days after cell transplantation. SDF-1 pretreatment exhibited a protective effect on ADSC survival. As shown in Fig. 4A, the number of surviving cells was significantly higher in the SDF-1 treatment group than in the apoptosis model group, suggesting that SDF-1 suppresses ADSC apoptosis in vivo.

SDF-1-pretreated ADSCs improve chronic wound healing in vivo. To further determine whether SDF-1 is able to promote the wound healing ability of ASDCs in diabetic mice, the healing of wounds was evaluated 10 days after treatment. The wound area was significantly smaller in the two groups treated with ADSCs than in the control group, and the wound closure index for SDF-1-pretreated ADSCs was significantly better than that in the other two groups (Fig. 4B). Histological results for hematoxylin and eosin staining of the wounds indicate that SDF-1-pretreated ADSCs can promote chronic wound repair in diabetic mice, as is shown by increased dermal collagen levels, fibroblast numbers and capillary density. The re-epithelization length in the SDF-1-pretreated ADSC group was significantly greater than that in the ADSC group and the control (Fig. 4C).

\section{Discussion}

In physiological cutaneous wound healing, MSCs are mobilized from host sources and localize to the site of injury, persisting to improve angiogenesis and support tissue repair and immunomodulation in cutaneous wounds (21). Despite somewhat challenging conditions in the wound bed, these host MSCs are able to perform normal functions. However, in the case of cell therapy where MSCs are administered to the wound bed, due to a lack of oxygen and nutrients and the effects of free radicals and inflammation, $>99 \%$ of the administered cells die following implantation $(7,14,22)$. Poor post-implant cell numbers often lead to the absence of more definitive results. Therefore, a significant barrier to the effective use of ADSCs in cell therapy has been the insufficient post-implant cell survival. As the benefits of ADSC therapy for cutaneous wound healing are dependent on cell survival in the wound bed, strategies to improve survival following implantation, such as hypoxic pretreatment (11) or genetic modification $(10,19)$ have received some attention. The present study identified for the first time, to the best of our knowledge, that SDF-1 pretreatment protects ADSCs from apoptosis in a hypoxic and serum-free environment. Furthermore, in vivo experiments established that the post-implant cell survival and chronic wound healing ability of ADSCs were increased following pretreatment with SDF-1 in a diabetic mouse model of chronic wound healing.

A number of studies have confirmed that SDF-1 enhances the proliferation, migration and secretion capacity of BMSCs and cardiac stem cells so as to improve the cell therapeutic effect in vivo $(16,17,23)$. For cell apoptosis, Yin et al found that SDF-1 inhibits hypoxia and serum deprivation-induced apoptosis in MSCs through phosphoinositide 3-kinase/Akt and extracellular signal-regulated kinase $1 / 2$ signaling pathways (24). Herberg et al demonstrated that SDF-1 mediates MSC survival through the enhancement of autophagy (14). Therefore, it was hypothesized that SDF-1 pretreatment may promote the activity and survival of ADSCs in an oxygen and nutrient-deficiency environment. In the present study, harsh ischemic microenvironment stress was mimicked by the use of serum-free and hypoxic conditions and these were found to induce marked apoptosis of ADSCs within $6 \mathrm{~h}$. Moreover, it was found that the apoptosis induced in hypoxic and serum-free conditions was ameliorated by SDF-1. Furthermore, the caspase-3 and PARP signaling pathway may be involved in the inhibition of cell apoptosis by SDF-1.

SDF-1 is a chemokine considered to play an important role in $\mathrm{CXCR}^{+}$and $\mathrm{CXCR}^{+}$progenitor cell mobilization and homing for ischemic or damaged tissues. It is secreted by endothelial and reticular cells and upregulated in acute cutaneous wounds for several days following trauma (25). SDF-1 upregulation in acute wounds takes part in the initial localization, retention and support of $\mathrm{CXCR}^{+}$stem cells, thus playing a critical role in physiological cutaneous wound healing (26). However, SDF-1 is expressed at low levels in chronic wounds, and may be hydrolytically inactivated by matrix metalloproteinases, leading to an SDF-1/CXCR4, CXCR7 axis disorder, hindering the migration and homing of cells and resulting in chronic healing or an absence of healing $(27,28)$. Therefore, in the cell therapy of chronic wounds, the wound environment becomes increasingly harsh, with excessive inflammation and an environment that is not conducive to angiogenesis $(29,30)$. The deficiency in the normal physiological process of cell mobilization and activation that SDF-1 induces leads to poor ADSC survival and homing. Therefore, it was hypothesized in the present study that pretreatment with SDF-1 may simulate the physiological process of cell mobilization to a certain extent and promote the therapeutic effect of ADSCs in cutaneous chronic wounds. Thus, ADSCs, either untreated or pretreated with $0.5 \mathrm{mg} / \mathrm{l}$ SDF-1, were administered by injection in a diabetic chronic wound model. It was found that the surviving cell number was significantly higher, and the wound closure index and length of re-epithelization were improved by SDF-1 pretreatment of the ADSCs, indicating a significant promoting effect of SDF-1 on the survival and therapeutic effect of ADSCs in vivo. 
In conclusion, the present study demonstrated that the chemokine SDF-1 is important for ADSC survival and the prevention of apoptosis. Pretreating ADSCs with SDF-1 prior to local administration or intravenous transplantation may significantly enhance cell survival and the therapeutic effect of ADSCs in cutaneous chronic wounds.

\section{Acknowledgements}

This study was supported by grants from the National Natural Science Foundation of China (grant nos. 81571901 and 81501671).

\section{References}

1. Montfrans CV, Stok M and Geerkens M: Biology of chronic wounds and new treatment strategies. Phlebology 29 (Suppl): 165-167, 2014.

2. Charbonneau L, Perrenoud B, Gallant S, Lehn I and Champier V: Chronic wound management in hospital care units - a literature review. Rech Soins Infirm 96: 58-68, 2009 (In French).

3. Fumimoto Y, Matsuyama A, Komoda H, et al: Creation of a rich subcutaneous vascular network with implanted adipose tissue-derived stromal cells and adipose tissue enhances subcutaneous grafting of islets in diabetic mice. Tissue Eng Part C Methods 15: 437-444, 2009.

4. Ohmura Y, Tanemura M, Kawaguchi N, et al: Combined transplantation of pancreatic islets and adipose tissue-derived stem cells enhances the survival and insulin function of islet grafts in diabetic mice. Transplantation 90: 1366-1373, 2010.

5. Zhu Y, Liu T, Song K, Fan X, Ma X and Cui Z: Adipose-derived stem cell: A better stem cell than BMSC. Cell Biochem Funct 26: 664-675, 2008.

6. Han C, Zhang L, Song L, Liu Y, Zou W, Piao H and Liu J: Human adipose-derived mesenchymal stem cells: A better cell source for nervous system regeneration. Chin Med J (Engl) 127: 329-337, 2014.

7. Nuschke A: Activity of mesenchymal stem cells in therapies for chronic skin wound healing. Organogenesis 10: 29-37, 2014.

8. Das R, Jahr H, van Osch GJ and Farrell E: The role of hypoxia in bone marrow-derived mesenchymal stem cells: Considerations for regenerative medicine approaches. Tissue Eng Part B Rev 16: $159-168,2010$.

9. Liu H, Xue W, Ge G, et al: Hypoxic preconditioning advances CXCR4 and CXCR7 expression by activating HIF-1 $\alpha$ in MSCs. Biochem Biophys Res Commun 401: 509-515, 2010.

10. Shevchenko EK, Makarevich PI, Tsokolaeva ZI, et al: Transplantation of modified human adipose derived stromal cells expressing VEGF165 results in more efficient angiogenic response in ischemic skeletal muscle. J Transl Med 11: 138, 2013.

11. Ruiz de Almodovar C, Luttun A and Carmeliet P: An SDF-1 trap for myeloid cells stimulates angiogenesis. Cell 124: 18-21, 2006.

12. Togel FE and Westenfelder C: Role of SDF-1 as a regulatory chemokine in renal regeneration after acute kidney injury. Kidney Int Suppl 1: 87-89, 2011.

13. Nakamura Y,Ishikawa H,Kawai K, Tabata Y and Suzuki S: Enhanced wound healing by topical administration of mesenchymal stem cells transfected with stromal cell-derived factor-1. Biomaterials 34 9393-9400, 2013.
14. Herberg S, Shi X, Johnson MH, Hamrick MW, Isales CM and Hill WD: Stromal cell-derived factor-1 $\beta$ mediates cell survival through enhancing autophagy in bone marrow-derived mesenchymal stem cells. PLoS One 8: e58207, 2013.

15. Liu H, Liu S, Li Y, et al: The role of SDF-1-CXCR4/CXCR7 axis in the therapeutic effects of hypoxia-preconditioned mesenchymal stem cells for renal ischemia/reperfusion injury. PLoS One 7: e34608, 2012.

16. Ghadge SK, Mühlstedt S, Ozcelik C and Bader M: SDF-1 $\alpha$ as a therapeutic stem cell homing factor in myocardial infarction. Pharmacol Ther 129: 97-108, 2011.

17. Wang K, Zhao X, Kuang C, et al: Overexpression of SDF-1 $\alpha$ enhanced migration and engraftment of cardiac stem cells and reduced infarcted size via CXCR4/PI3K pathway. PLoS One 7: e43922, 2012.

18. Li Q, Zhang A, Tao C, Li X and Jin P: The role of SDF-1-CXCR4/CXCR7 axis in biological behaviors of adipose tissue-derived mesenchymal stem cells in vitro. Biochem Biophys Res Commun 441: 675-680, 2013.

19. Li Q, Li PH, Hou DJ, et al: EGF enhances ADSCs secretion via ERK and JNK pathways. Cell Biochem Biophys 69: 189-196, 2014.

20. Martínez-Santamaría L, Conti CJ, Llames S, et al: The regenerative potential of fibroblasts in a new diabetes-induced delayed humanised wound healing model. Exp Dermatol 22: 195-201, 2013.

21. Li H and Fu X: Mechanisms of action of mesenchymal stem cells in cutaneous wound repair and regeneration. Cell Tissue Res 348: 371-377, 2012.

22. Gojo S, Gojo N, Takeda Y, et al: In vivo cardiovasculogenesis by direct injection of isolated adult mesenchymal stem cells. Exp Cell Res 288: 51-59, 2003.

23. Liu X, Zuo D, Fan H, et al: Over-expression of CXCR4 on mesenchymal stem cells protect against experimental colitis via immunomodulatory functions in impaired tissue. J Mol Histol 45: 181-193, 2014.

24. Yin Q, Jin P, Liu X, et al: SDF-1 $\alpha$ inhibits hypoxia and serum deprivation-induced apoptosis in mesenchymal stem cells through PI3K/Akt and ERK1/2 signaling pathways. Mol Biol Rep 38: 9-16, 2011.

25. Zong ZW, Cheng TM, Su YP, et al: Crucial role of SDF-1/CXCR4 interaction in the recruitment of transplanted dermal multipotent cells to sublethally irradiated bone marrow. J Radiat Res (Tokyo) 47: 287-293, 2006.

26. Askari AT, Unzek S, Popovic ZB, et al: Effect of stromal-cell-derived factor 1 on stem-cell homing and tissue regeneration in ischaemic cardiomyopathy. Lancet 362: 697-703, 2003.

27. Brem $\mathrm{H}$ and Tomic-Canic M: Cellular and molecular basis of wound healing in diabetes. J Clin Invest 117: 1219-1222, 2007.

28. Litwiniuk M, Bikowska B, Niderla-Bielińska J, et al: Potential role of metalloproteinase inhibitors from radiation-sterilized amnion dressings in the healing of venous leg ulcers. Mol Med Rep 6: 723-728, 2012.

29. Khanna S, Biswas S, Shang Y, et al: Macrophage dysfunction impairs resolution of inflammation in the wounds of diabetic mice. PLoS ONE 5: e9539, 2010.

30. Krisp C, Jacobsen F, McKay MJ, Molloy MP, Steinstraesser L and Wolters DA: Proteome analysis reveals antiangiogenic environments in chronic wounds of diabetes mellitus type 2 patients. Proteomics 13: 2670-2681, 2013. 